\title{
Desempenho ocupacional e estresse: aplicação de manual de orientações e cuidados a gestantes de risco*
}

\section{Occupational performance and stress: application of a manual for guidance and care of high-risk pregnant women}

\author{
Janaína Armendaris Cruz ${ }^{1}$, Nicole Ruas Guarany ${ }^{2}$
}

http://dx.doi.org/10.11606/issn.2238-6149.v26i2p201-206

Cruz JA, Guarany NR. Desempenho ocupacional e estresse: aplicação de manual de orientações e cuidados a gestantes de risco. Rev Ter Ocup Univ São Paulo. 2015 maio-ago.;26(2):201-6.

RESUMO: A gestação é um fenômeno biologicamente natural e é considerada de alto risco quando há maior perigo para a saúde da mãe e do feto, devido a alguma doença materna. $\mathrm{O}$ objetivo deste trabalho foi desenvolver e aplicar um manual de orientações e cuidados às gestantes de alto risco, identificando sua influência no desempenho ocupacional e estresse. Consiste em estudo transversal, de acompanhamento com avaliação antes e depois. Utilizou-se a Medida Canadense de Desempenho Ocupacional (COPM) e a Escala de Estresse Percebido (PPS-10). A amostra do estudo foi composta por onze gestantes de alto risco internadas. A COPM identificou 2,9 pontos para a área de Desempenho e 2,7 pontos para área de Satisfação com o desempenho na primeira avaliação e 4 pontos e 4,2, respectivamente, na segunda avaliação após a aplicação do Manual de Orientações. A PPS-10 indicou 21 pontos na primeira avaliação e 17,9 pontos na reavaliação. Nesse sentido, acredita-se que grupos de apoio às gestantes, estabelecendo espaços de diálogo e escuta, permitem a construção de alternativas para enfrentamento das dificuldades advindas da internação.

DESCRITORES: Gravidez de alto risco; Questionários; Terapia ocupacional.
Cruz JA, Guarany NR. Occupational performance and stress: application of a manual for guidance and care of high-risk pregnant women. Rev Ter Ocup Univ São Paulo. 2015 May-Aug.;26(2):201-6.

ABSTRACT: Gestation is a natural biological phenomenon, and it is considered of high-risk when there is increased danger for the health of the mother and fetus due to some maternal disease. The purpose of this work was to develop and implement a manual for guidance and care of high-risk pregnant women, identifying its influence on occupational performance and stress. This is a cross-sectional study whit follow-up evaluation before and after. The Canadian Occupational Performance Measure (COPM) and the Perceived Stress Scale (PPS-10) were used. The study sample comprised 11 inpatient high-risk pregnant women. The COPM identified 2.9 points for the Performance area and 2.7 points for the Performance satisfaction area in the first evaluation, and 4 and 4.2 points, respectively, in the second evaluation after the application of the Guidance Manual. The PPS-10 indicated 21 points in the first evaluation and 17.9 points in the revaluation. In this context, it is believed that support groups for pregnant women, establishing spaces for dialogue and listening, allow the construction of alternatives for facing the difficulties resulted from hospitalization.

KEYWORDS: Pregnancy, high-risk; Questionnaires; Occupational therapy.

\footnotetext{
* Resultado do Trabalho de Conclusão de Curso (TCC) do Curso de Terapia Ocupacional da Universidade Federal de Pelotas, Rio Grande do Sul.

1. Acadêmica de Terapia Ocupacional da Universidade Federal de Pelotas, Rio Grande do Sul.

2. Mestre em Saúde da Criança e do Adolescente, docente do curso de Terapia Ocupacional da Universidade Federal de Pelotas, Rio Grande do Sul.

Endereço para correspondência: Rua Professor João de Souza Ribeiro, 155/719. Bairro Humaitá. Porto Alegre, RS. E-mail: janacz@ hotmail.com.
} 


\section{INTRODUÇÃO}

\section{A} gestação é um evento fisiológico na vida de uma mulher, que ocasiona diferentes transformações, gerando mudanças físicas e emocionais relacionadas a esse estado. Na maioria dos casos a gravidez tem sua evolução normal, sem complicações. Existe um pequeno grupo de gestantes que, por características específicas ou por sofrerem algum agravo ao longo da gestação, apresentam maior possibilidade de evolução desfavorável, sujeita a complicações que podem afetar tanto o feto como a mãe. Essas gestantes são consideradas de alto risco ${ }^{1}$.

Há vários fatores causadores de risco gestacional relacionados a características individuais, fatores socioeconômicos e demográficos desfavoráveis, história obstétrica ou reprodutiva, situação clínica materna, doença obstétrica atual, intercorrências clínicas e hábitos durante a gestação (fumo, álcool, drogas) ${ }^{2}$. Tais fatores influenciam diretamente no cotidiano das gestantes e podem ocasionar longos períodos de internação e, assim, impactar nas tarefas cotidianas de desempenho ocupacional.

O desempenho ocupacional refere-se ao ato de fazer e a capacidade de realizar uma atividade ou ocupação que resulta da combinação dinâmica entre o indivíduo, o contexto e a atividade. Fornece ou capacita habilidades e padrões de desempenho ocupacional, conduzindo a um envolvimento em ocupações ou atividades apropriadas para o estágio de desenvolvimento, cultura e ambiente do indivíduo. Os papéis ocupacionais desenvolvem-se em conjunto com as ocupações que o indivíduo desempenha na sociedade ${ }^{3}$. Os papéis ocupacionais de gestante e futura mãe podem ser rompidos quando a gestação se torna de risco, e, muitas vezes, ao longo do período de hospitalização, deixa essas mulheres mais suscetíveis a fatores estressantes. Este é o momento em que a grávida se conscientiza em relação a sua situação singular, que pode acarretar em afastamento do suporte familiar e na vivência de conflitos entre a dependência necessária e a perda de autonomia. $\mathrm{O}$ estresse consiste em alguma situação na qual o equilíbrio homeostático do corpo é perturbado, promovendo adaptações orgânicas como a liberação de grande quantidade de hormônios na corrente sanguínea, preparando o indivíduo para algum tipo de reação ${ }^{4}$. O período gestacional é considerado um estado específico de estresse emocional ${ }^{5,6}$ por envolver intensas mudanças na vida da mulher sob o ponto de vista físico, psicológico e social. O corpo em constante transformação, a instabilidade emocional, o medo e a ansiedade gerados pelo parto, a transição do papel social de filha e mulher para mãe e outros fatores do cotidiano, influenciam a saúde psicológica da gestante, predispondo-a, em maior ou menor grau, ao estresse ${ }^{7}$.

A Terapia Ocupacional é fundamentada na compreensão de que o envolvimento em ocupações estrutura a vida cotidiana e contribui para a saúde e para o bem-estar. $\mathrm{O}$ envolvimento na ocupação envolve ambos aspectos de desempenho: os subjetivos (emocionais e psicológicos) e os objetivos (fisicamente observáveis). Dessa maneira dar-se-á o entendimento do envolvimento a partir dessa perspectiva dual e holística, recorrendo a todos os aspectos do desempenho quando fornecer a intervenção ${ }^{3}$.

Encontram-se na literatura inúmeras publicações referentes ao cuidado, prevenção e assistência às gestantes de alto risco em diferentes áreas e equipes profissionais, porém ainda são escassas pesquisas que demonstram o impacto que essas intercorrências causam no desempenho ocupacional e a influência do estresse nessas situações. Desta forma, pensando em demonstrar o quanto o papel do terapeuta ocupacional é de fundamental importância nessas situações e como pode influenciar de maneira positiva por meio de uma intervenção terapêutica adequada, o objetivo deste trabalho foi desenvolver e aplicar um manual de orientações e cuidados às gestantes de alto risco, identificando sua influência no desempenho ocupacional e estresse.

\section{PROCEDIMENTOS METODOLÓGICOS}

Estudo do tipo transversal, de acompanhamento com avaliação antes e depois, de caráter qualitativo quanto à aplicação do manual de orientações às gestantes e quantitativo de acordo com a análise de dados referentes aos instrumentos utilizados. Foram convidadas a participar do estudo, por meio de amostragem por conveniência, todas as gestantes com gravidez de risco identificadas por avaliação médica (intercorrências clínicas ou alguma doença diagnosticada, de qualquer idade), que estivessem internadas na Casa da Gestante do Hospital São Francisco de Paula, na cidade de Pelotas, vinculada à Universidade Católica de Pelotas, entre os meses de abril e maio de 2014, com tempo mínimo de internação de 7 dias e alfabetizadas, sendo incluídas após assinatura do Termo de Consentimento Livre e Esclarecido. Foram excluídas do estudo as gestantes com retardo mental.

Os dados sociodemográficos, o histórico gestacional e a saúde da gestante foram avaliados por meio de questionário específico, construído pelos autores. O Manual de Orientações às Gestantes de Risco foi feito por meio de revisão bibliográfica sobre os problemas de 
saúde mais comuns durante a gestação que levavam à internação hospitalar, sobre autocuidado, orientações de posicionamento da gestante, além de cuidados com o bebê. As atividades de aplicação foram abordadas por esclarecimentos e atividades didáticas e realizadas no espaço de convivência do local, com duração de duas horas no período de quatro dias contínuos. As atividades foram dividas da seguinte forma: primeiro encontro Terapia Ocupacional e a gestante, cuidado materno e posicionamento; segundo encontro - Cuidado com o bebê e amamentação; terceiro encontro - Desenvolvimento neuropsicomotor do bebê.

O desempenho ocupacional foi avaliado por meio da Medida Canadense de Desempenho Ocupacional (COMP), que engloba as áreas de autocuidado, produtividade e lazer ${ }^{8}$. Para a avaliação de estresse foi utilizada a Escala de Estresse Percebido (PPS - 10) validada para o português. As questões versam a respeito dos sentimentos e pensamentos das gestantes durante os trinta dias que precedem o questionário e o instrumento indica que quanto mais alta a pontuação, maior o nível de estresse $\mathrm{e}^{9,10}$.

Esta pesquisa foi submetida à Plataforma Brasil e aprovada pelo Comitê de Ética e Pesquisa da Universidade Federal de Pelotas, sob $\mathrm{n}^{\circ}$ 579.947/05/03/2014 e da Universidade Católica de Pelotas, responsável pelo local de realização da pesquisa.

As variáveis qualitativas foram descritas na forma de frequência simples e as quantitativas na forma medianas e intervalos interquartis. A comparação entre os resultados dos instrumentos nos dois momentos do estudo (antes e após a aplicação do manual de orientações) foi realizada pelo teste de Wilcoxon. O nível de significância foi de $\mathrm{p}<0,05$.

\section{RESULTADOS E DISCUSSÃO}

A amostra do estudo foi composta por onze gestantes de alto risco internadas na Casa da Gestante do Hospital São Francisco de Paula, no período de maio de 2014. A idade das gestantes variava entre 18 e 39 anos, a maior parte trabalhava fora de casa $(n=6)$ e encontrava-se afastada do emprego devido às internações hospitalares. No que tange ao item escolaridade, apenas duas haviam completado o ensino médio e possuíam renda entre $\mathrm{R} \$ 900$ e R \$1000. A maioria das gestantes contava com a presença de marido/companheiro ao longo da gestação $(n=9)$.

Segundo Rezende ${ }^{11}$, a família é de grande importância quando inserida no contexto da hospitalização, contribuindo para um suporte familiar, dividindo as responsabilidades do lar (cuidando dos filhos, serviços domésticos, assessorando o lar de uma forma geral), o que faz as gestantes sentirem-se mais seguras e tranquilas, podendo enfrentar melhor sua internação, sem preocuparse com o afastamento de seu lar.

Em relação às características gestacionais, apenas três gestantes alegaram que suas gestações haviam sido planejadas e nove relataram já possuir outros filhos, sendo que, destas, cinco estavam à espera do quarto filho. Em relação às intercorrências em gestações anteriores, sete confirmaram problemas. $\mathrm{Na}$ amostra geral, os motivos de internação e complicações variavam, porém o mais comumente apresentado foi a diabetes gestacional $(n=4)$.

A diabetes gestacional é definida como a intolerância aos carboidratos, de graus variados de intensidade, diagnosticada pela primeira vez durante a gestação, podendo ou não persistir após o parto. No Brasil, a prevalência da diabetes gestacional em mulheres com mais de 20 anos, atendidas no Sistema Único de Saúde, é de 7,6\%,94\% dos casos apresentam apenas tolerância diminuída à glicose e $6 \%$ indicam hiperglicemia no nível de diabetes fora da gravidez ${ }^{1}$. Outro importante fator determinante de internações, que também foi encontrado em $(n=3)$ dos casos, é o trabalho de parto prematuro (TPP), responsável por $75 \%$ dos nascimentos antes da $37^{\mathrm{a}}$ semana de gestação. Sua prevenção durante o pré-natal é poucas vezes possível, pois, geralmente, apresenta etiologia multifatorial ou desconhecida ${ }^{12}$.

O resultado do teste de Wilcoxon não encontrou diferenças para a avaliação de estresse por meio do PPS10 antes e depois da aplicação do Manual de orientações para gestantes $(\mathrm{p}=0,97)$ e para a análise do desempenho ocupacional pelo COMP na área de Desempenho $(\mathrm{p}=0,138)$ e na área de Satisfação $(p=0,176)$.

A escala de estresse (PPS-10) identificou 21,09 pontos na primeira avaliação e 17,9 pontos na reavaliação após a aplicação do Manual de orientações. A maioria das gestantes apresentou maiores níveis de estresse, comparadas a estudos com populações diversas, que mostraram valores de estresse de 14 a $21^{9,10}$, assim como achados no estudo de Segato ${ }^{7}$, realizado com gestantes utilizando o mesmo instrumento de mensuração de estresse, em que a maioria das gestantes $(93 \%)$ manifestou elevados níveis de estresse percebido ( 28,8 pontos).

Estudos apontam para a existência de grande número de mulheres com níveis significativos de ansiedade durante a gestação, manifestada, sobretudo, no terceiro trimestre gestacional com a proximidade do parto $^{13}$, indo de encontro aos resultados dessa pesquisa, em que $(n=8)$ das gestantes encontravam-se no terceiro 
trimestre da gestação. A internação pode influenciar nos ajustes emocionais, sociais e físicos impostos pela gravidez, causando níveis variados de estresse e ansiedade individual e familiar ${ }^{14}$. Não foram verificadas diferenças significativas nos níveis de estresse das gestantes pré e pós atividades grupais do manual, no entanto observou-se redução do nível de estresse em 4 pontos. Acredita-se que em um período mais longo de intervenções o resultado poderia ser modificado positivamente.

Os efeitos esperados com a utilização do Manual de Orientações às Gestantes como meio intermediador e a elaboração de atividades grupais são os de proporcionar à gestante a oportunidade de discutir algumas de suas ansiedades, podendo ajudar também na prevenção de complicações, ações educativas e problemas relacionados ao desempenho de atividades cotidianas. Dessa forma, proporciona-se a mudança do foco de atenção para pensamentos que conduzam a uma sensação de tranquilidade (o bebê), promovendo a qualidade de saúde no processo de hospitalização. Tendo em vista que as ações coletivas em grupo possibilitam o desenvolvimento natural de um espaço para o movimento da promoção da saúde por meio de um processo de ensino-aprendizagem, pode-se constituir num método privilegiado de intervenção do profissional de Terapia Ocupacional ${ }^{15}$.

Segundo o Ministério da Saúde ${ }^{1}$, é importante destacar uma abordagem integral às mulheres, considerando-se as especificidades relacionadas às questões de gênero, raça, etnia, classe social, escolaridade, situação conjugal e familiar, trabalho, renda e atividades laborais, entre outras. Essa atenção implica a valorização de práticas que privilegiem a escuta e a compreensão sobre os diversos fenômenos que determinam maior ou menor condição de risco à gestação. $\mathrm{O}$ acolhimento da gestante por toda a equipe de saúde, além de qualificar a assistência, possibilitará o estabelecimento de vínculos, maior responsabilização pelo processo de cuidado e o manejo sobre situações de vulnerabilidade relacionadas ao processo saúde-doença, sejam elas individuais, sociais e até mesmo programáticas.

$\mathrm{Na}$ avaliação de desempenho ocupacional, a COMP na primeira avaliação apresentou 2,9 pontos na área de Desempenho e na reavaliação, 4 pontos. $\mathrm{Na}$ área de Satisfação a primeira apresentou 2,7 pontos e após a reavaliação, 4,2 pontos.

As atividades mais frequentemente elucidadas pelas gestantes como problemas de desempenho ocupacional, a partir da COMP, foram "limpar/arrumar a casa" e "cuidar dos filhos", corroborando com estudo de Costa e Silva ${ }^{16}$ realizado com gestantes, também hospitalizadas, no qual as mesmas comentaram a modificação de seus papéis sociais, devido à gestação de alto risco e ao processo de hospitalização, deixando clara a quebra de seus papéis sociais de esposa e de mãe, retratando o afastamento do domicílio, de suas atividades e do companheiro, inibindo sua qualidade de vida e de sua satisfação pessoal.

Hagedorn ${ }^{17}$ define papel social como:

"[...] aquele que designa a relação, a responsabilidade ou o status dentro de uma cultura ou grupo que dirige o envolvimento do indivíduo em certas ocupações, atividades ou tarefas relacionadas ao papel por longos períodos".

Ao considerar o desempenho ocupacional como o campo de atuação do terapeuta ocupacional, Magalhães e Oliveira ${ }^{18}$ têm a atividade humana como um processo criativo, criador, lúdico, expressivo, evolutivo, produtivo e de automanutenção. Adicionalmente, concebem o homem como um ser práxico, e a ação do terapeuta ocupacional interfere em seu cotidiano com a finalidade de proporcionar-lhe qualidade de vida, respeitando sua completude, considerando seu estado psicológico, social, laborativo e de lazer, entre outros.

Nesse contexto, em que a mulher vivencia situações não comumente habituais de uma gestação normal, a Terapia Ocupacional tem como objetivo intervir por meio do reconhecimento de experiências prazerosas e de possibilidades remanescentes que, no ambiente em que estão inseridas, podem trazer benefícios para o bem-estar físico, mental e psicológico, e, assim, promover a melhora do desempenho ocupacional e do estresse.

No momento da reavaliação e do encerramento das atividades, os discursos das gestantes versavam quanto à satisfação em terem a oportunidade de explicitar dúvidas e estreitar laços entre elas. Corroboram, dessa forma, com os resultados encontrados em um estudo realizado com um grupo de mães de Minas Gerais, no qual reconhece-se a importância da criação de espaços onde as mães possam expressar as demandas relacionadas a si mesmas e ao momento vivido, favorecendo a diminuição do estresse psicológico durante a internação e a formação de uma rede de apoio com outras mães que vivenciam a mesma situação e dessas com os profissionais de saúde como meio socializante, promovem a construção de laços afetivos e fortalecem as relações de amizade entre as mães que experienciam a mesma situação ${ }^{19}$. 


\section{CONCLUSÃO}

A gravidez é um momento importante e marcante na vida de uma mulher; sendo assim, as gestantes que passam pelo processo de hospitalização atentam para um cuidado especializado. Os resultados do estudo demonstram o quanto o distanciamento de casa influencia no desempenho ocupacional e no estresse dessas gestantes. Com as atividades realizadas por meio do Manual de Orientações para a Gestante, foi possível promover discussões e conhecimentos em relação à educação em saúde, ao autocuidado e cuidado com o bebê, além de proporcionar um espaço para convivência entre as gestantes, diminuindo o impacto da hospitalização.

\section{REFERÊNCIAS}

1. Brasil. Ministério da Saúde. Secretaria de Atenção à Saúde. Departamento de Ações Programáticas Estratégicas. Gestação de alto risco: manual técnico. 5a ed. Brasília, DF: Editora do Ministério da Saúde; 2012.

2. Costa IG. As percepções da gravidez de risco para a gestante e as implicações familiares. Rev Gaúcha Enferm (Porto Alegre). 2002;23(1):30-46. Disponível em: file://C:/Users/ FMUSP/Downloads/4391-14115-1-PB.pdf;

3. AOTA. Occupational Therapy Practice. Framework: domain \& process. 2nd. Am J Occup Ther. 2008;63(6):625-83. Available from: http:/www.polk.edu/wp-content/uploads/ OTFramework2ndEdition.pdf.

4. Margis R; Picon P; Cosner AF; Silveira RO. Relação entre estressores, estresse e ansiedade. Rev Psiquiatr (Porto Alegre). 2003;25(Supl 1):65-74. DOI: http://dx.doi. org/10.1590/S0101-81082003000400008

5. Huizink AC, Medina PGR, Mulder EJH, Visser GHA, Buitelaar JK. Psychological measures of prenatal stress as predictors of infant temperament. J Am Acad Child Adolesc Psychiatr. 2002;41(9):1078-85. Available from: http://www. sciencedirect.com/science/journal/08908567/41/9

6. Bjelica A. Pregnancy as a stressful life event and strategies for coping with stress in women with pregnancy induced hypertension. Med Pregled Novi Sad. 2004;57(78):367-8. Disponível em: http://www.ncbi.nlm.nih.gov/ pubmed/15626294

7. Segato L, Vasconcelos DIC, Rolim MKSB, Andrade A, Matias TS. Ocorrência e controle do estresse em gestantes sedentárias e fisicamente ativas. Rev Educ Físi UEM
Pontuam-se como limitações do estudo a dificuldade em captar um número amostral maior, devido à não-permanência das gestantes por um período prolongado de tempo no local de pesquisa, e a recusa das gestantes em participar do estudo em razão de condições clínicas.

Embora as avaliações pré e pós-aplicação do Manual de Orientações para Gestantes não tenham encontrado diferença significativamente estatística para estresse e desempenho ocupacional, considera-se que a intervenção foi válida, visto a troca de experiências e abrangência do campo da Terapia Ocupacional também para um cuidado especial com essa população. Além disso, os resultados podem servir de ponto de partida para estudos futuros, nos quais possam ser indicadas atividades e intervenções para esse público específico.

(Maringá). 2009;20(1):121-9. DOI: 10.4025/reveducfis. v20i1.6062.

8. Earlyb MB. Desempenho ocupacional. In: Pedretti LW, Eaerly MB. Terapia ocupacional: capacidades práticas para as disfunções físicas. São Paulo: Roca; 2005. p.125-31.

9. Cohen S, Kamarck T, Mermelstein R. A global measure of perceived stress. J Health Social Behav. 1983;24(4):385-96. Availalble from: http://www.jstor.org/stable/pdf/2136404. pdf?\&acceptTC=true \&jpdConfirm=true.

10. Reis RS, HinoA, Rodriguez-Añez CR. Perceived stress scale: reliability and validity study in Brazil. J Health Psychol. 2010;15(1):107-14. DOI:10.1177/1359105309346343.

11. Rezende CL. Qualidade de vida das gestantes de alto risco em Centro de Atendimento à Mulher do município de Dourados, MS. [Dissertação]. Campo Grande: Universidade Católica Dom Bosco; 2012.

12. Bittar RE, Zugaib M. Indicadores de risco para o parto prematuro. Rev Bras Ginecol Obstet [online]. 2009;31(4):203-9. DOI: http://dx.doi.org/10.1590/S010072032009000400008 .

13. Araújo DM, Pererira NL, Kac G. Ansiedade na gestação, prematuridade e baixo peso ao nascer: uma revisão sistemática da literatura. Cad Saúde Pública (Rio de Janeiro). 2007;23(4):747-56. DOI: http://dx.doi.org/10.1590/S0102311X2007000400002.

14. Araújo MA, Albertini R, Guimarães FP. Incidência de sintomas de estresse em primíparas: vivências e relatos de mulheres. Polêm!ca. 2010;9(4):64-73. Disponível em: http://www.epublicacoes.uerj.br/index.php/polemica/article/view/2821. 
Cruz JA, Guarany NR. Desempenho ocupacional e estresse. Rev Ter Ocup Univ São Paulo. 2015 maio/ago.;26(2):201-6.

15. Delfino MRR, Patrício MZ, Martins A, Silvério MR. O processo de cuidar participante com um grupo de gestantes: repercussões na saúde integral individual-coletiva. Ciên Saúde Coletiva. 2004;9(4):1057-66. DOI: http://dx.doi. org/10.1590/S1413-81232004000400026.

16. Costa e Silva MR, Vieira BDG,Alves VH, Rodrigues DP, Vargas GS, Sá AMP. A percepção de gestantes de alto risco acerca do processo de hospitalização. Rev Enferm UERJ (Rio de Janeiro). 2013;21(esp. 2):792-7. Disponível em: http:// www.e-publicacoes.uerj.br/index.php/enfermagemuerj/ article/view/12295

Recebido para publicação: 29.10.14

Aceito para publicação: 16.06 .15
17. Hagedorn R. Fundamentos para a prática em terapia ocupacional. São Paulo: ROCA; 2003.

18. Magalhães DF, Oliveira CMA. Atenção básica de saúde e as perspectivas políticas profissionais da Terapia Ocupacional. Rev Baiana Saúde Pública.2008;32(supl. 1):72-80. Disponível em: http://inseer.ibict.br/rbsp/index. $\mathrm{php} / \mathrm{rbsp} /$ article/viewFile/1460/1096

19. Duarte ED, Dittz ES, Silva BCN, Rocha LLB. Grupos de apoio às mães de recém-nascidos internados em unidade neonatal. Rev Rene. 2013;14(3):630-8. Disponível em: file://C:/Users/FMUSP/Downloads/1397-7049-1-PB.pdf. 